\title{
Determining the Representative Volume Element Size for Three-Dimensional Microstructural Material Characterization. Part 1: Predictive Models
}

\author{
William Harris and Wilson K. S. Chiu* \\ Department of Mechanical Engineering \\ University of Connecticut \\ 191 Auditorium Road \\ Storrs, CT 06269-3139 \\ * Corresponding Author, E-mail: wchiu@engr.uconn.edu.
}

\begin{abstract}
Recent advances in microstructural imaging and characterization tools have led to an increase in the direct examination of 3-D microstructures of composite materials in devices such as solid oxide fuel cells, battery electrodes, and composite gas separation membranes. In particular, focused ion beam-scanning electron microscopy and x-ray nanotomography methods have opened the door for materials characterization at the tens of nanometer scale. These experimental methods have enabled the quantitative evaluation of microstructures for a variety of important parameters. However, there is often uncertainty in the literature regarding the necessary size of a representative volume element (RVE) in order to capture an accurate description of the structure. In an effort to provide guidance for the field, in this work simplified geometric models are presented to provide basis for the a priori estimation of RVE size for three common microstructural parameters: volume fraction, particle size, and network contiguity. In a second and complementary paper (Part 2), the results of the model will be compared to experimental data obtained from the extensive imaging of a multiphase composite membrane material using x-ray nanotomography.
\end{abstract}

1. Introduction to the RVE concept 
In the study of material microstructures, the characterization of a large material domain can often be simplified by considering a smaller sub-section of the material termed a representative volume element (RVE). The RVE is used to link the heterogeneities at the microscale with effective parameters used to describe the material at the macroscale. The motivation to study materials via an RVE is that it permits smaller computational and experimental domains while still maintaining a description of material behavior relevant to device performance. The RVE concept has been used extensively in the field of continuum mechanics to describe the stress and strain fields within composite materials.[1-7] These studies are typically concerned with predicting the effective physical properties of the material under mechanical load, namely the elastic modulus. However, with recent developments in high resolution 3-D imaging capabilities, the RVE concept has been extensively applied in terms of accurate characterization, as well as simulation, of morphological properties in real physical systems. In particular, focused ion beam-scanning electron microscopy (FIB-SEM) and x-ray nanotomography methods capable of resolving the detailed distribution of multiple solid phases at the nanometer scale have helped move towards bridging the gap of understanding the connections between microscale material features and macroscale device performance.[8-12]

Several researchers have explicitly considered the effect of the imaging volume size on characterization results.[13-21] From a characterization perspective, the goal of the experimentalist is to sample just a small portion of a total material domain, and in considering just this small region, be able to generate a description that will represent the whole material. For this to be possible, an RVE extracted from any location within the material must statistically contain approximately the same structure and properties as any other randomly selected RVE. This requires the RVE to include a sufficient sampling of the variety of microstructural features 
that exist in the sample, such that a smaller sub-volume will contain an inadequate sampling of these features and therefore not be representative, while a larger sub-volume will contain more than a sufficient number of features and therefore will also be statistically representative. In that light, we seek a minimum RVE size, understanding that larger sub-volumes can also be used as an RVE. Implicit in this definition is the assumption that the bulk material displays a level of statistical homogeneity at some length scale. In other words, on a local scale the material may have a variety of heterogeneous structures, phases, and complex geometries that are arranged in a specific way unique to that exact location, but on a statistical basis the effective properties of the multiphase material are uniform across a much larger length scale. This would, for example, exclude graded structures in which a property such as particle size or volume fraction has been intentionally varied across the structure during the fabrication process.

In this study, for the sake of uniformity and general applicability, sub-volumes extracted from a total material domain will be restricted to cubic geometries. This is done to remove some of the ambiguity involved in defining RVE sizes based purely on volume, in which case it is not clear over what range of geometry (aspect ratio and shape) that definition is applicable. It is realized that the measurement of some properties depends solely on a count of some feature, such as the number of particles (and therefore volume fraction) of a given phase. For these types of "counting" property measurements, only the total volume that is analyzed, and not the volume's geometry, is significant. However, for some property measurements, particularly those aimed at describing pathway-type transport, the characterization results will almost certainly not be the same for two RVE's with very different aspect ratios even if the total volume is the same due to the influence of the boundaries. Therefore, the cube has been chosen here as the standard shape, realizing that that restriction could be relaxed in some instances if desired. 
The challenge to the experimentalist is that when imaging is to be performed on a given sample, the minimum RVE size to obtain accurate characterization results is generally not known a priori. Furthermore, the necessary size of an RVE may vary depending on the material property and level of precision in which the researcher is interested. In the literature, typically 3$\mathrm{D}$ image data is therefore analyzed after imaging to assess if volume-independent conditions have been met. In an effort to contribute to some predictive capabilities, this work outlines simplified models aimed at estimating the minimum RVE size for several common microstructural properties: volume fraction, phase size distribution, and network contiguity. In Part 2 of this work, the models are compared to experimental results obtained through extensive x-ray nanotomography imaging of a multiphase composite oxygen separation membrane.

\section{Predictive Models}

The models presented in the following sections use geometric simplifications of the complex and random microstructures that are often found in real materials. The simplified systems allow the variations in the structure to be described with a statistical basis in a tractable manner, with the understanding that the results serve only as approximations for real microstructures. Specifically, the models are designed for composite systems consisting of two or more phases (with pores treated equivalently to a solid phase), composed of randomly distributed particle-like features of relatively uniform size and exhibiting long-range statistical homogeneity. This type of description applies to a number of materials which are fabricated by the mixing and pressing of various powders, followed by sintering at high temperature. During the sintering process, the powders densify to form the particle-like features considered in this work. Numerous researchers have also used a simulation approach to generate material 
microstructures by random packing of spherical particles within a volume, sometimes followed by a simulated sintering process.[19-22] It is realized that for most real materials, the concept of a "particle" is an abstraction, as neighboring features which are in close contact frequently form a contiguous network rather than an array of isolated particles. Understanding this distinction, it is assumed that these complex structures can still be represented approximately as an assembly of particles, some of which are in close contact with their neighbors due to the sintering process. For phases forming a contiguous structure, the terms "particle size" and "feature size" are thus used interchangeably. In addition to the references mentioned above, the idealization of real microstructures as random arrangements of particles of various phases has been used numerous times in the literature to develop analytical descriptions of device performance.[23-26] Certain features which may exist in real materials are not covered in this framework, including possible non-random agglomeration of particles of a certain phase, large disparities in structural/particle length scales, highly anisotropic features such as fibrous or planar structures, and graded structures. Therefore, the descriptions presented are not universally applicable, but rather apply to a certain class of particle-like materials. As a cubic RVE size is sought for each property, we introduce a convenient way to scale the size of the cube

$$
L^{*}=\frac{\text { CubeEdgeLength }[\mu m]}{\text { ParticleSi ze }[\mu m]}
$$

The parameter $\mathrm{L}^{*}$ provides a measure of the size of a cube by using the average number of particles per side as the measuring unit. The measure of average particle size is an approximation made by the researcher, and is meant to be a characteristic length of all particles/features in the microstructure.

2.1. Analytical Model and Results for the Estimation of Volume Fraction RVE Size 
In this model, particles of various phases are assumed to be of constant size, randomlylocated, independent, and densely packed. In reality, close contact and sintering between neighboring particles may create interconnections of somewhat smaller size than the "particles" themselves. However, in the evaluation of volume fraction, it is acknowledged that most of the material volume of a given phase is contained in the "particles" themselves rather than these tenuous connections. Therefore, sintered regions of varying (smaller) size than the constituent particles are not explicitly considered. While these are substantial simplifications, they allow for the development of a simple model when little is known about a real material a priori.

Considering one phase at a time, a certain fraction $p$ of the particles belong to the phase of interest, and any such particle belonging to that phase is denoted as a "success". Given $p$ and a sampling of $n$ random and independent particles, the probability of observing a given number of successes $n_{i}$ follows the binomial distribution. For a real material, the volume fraction of the phase, $p$, is not known a priori. In fact, it is the volume fraction itself which the researcher is trying to accurately determine via an RVE. However, in this formulation $p$ can be treated as a researcher's estimate of the volume fraction. The estimate can be provided based on known fabrication conditions or preliminary pilot studies of the material, for example with limited 2D imaging and stereology techniques.

If $n$ total particles are sampled with a probability $p$ of success for any given particle, the mean number of successes is given by

$$
\mu=n_{i}=n p
$$

and if sampling of $n$ total particles is repeated for many trials, the standard deviation of the number of successes is

$$
\sigma=\sqrt{n p(1-p)}
$$


The mean and the standard deviation in Eqs. 2 and 3 are both in terms of the number of particles. However, to compute the volume fraction $\phi$ of a phase, we are interested in the fraction of particles which belong to that phase. (The variable $p$ is used to represent a researcher's a priori estimate of volume fraction, while $\phi$ denotes the sought-after true volume fraction.) We therefore divide by the total number of particles $n$ to obtain the expected mean $\mu_{\square}$ and standard deviation $\sigma_{\square}$ of the volume fraction of the phase of interest.

$$
\begin{aligned}
& \mu_{\phi}=\frac{\mu}{n}=p \\
& \sigma_{\phi}=\sigma / n=\sqrt{\frac{p(1-p)}{n}}
\end{aligned}
$$

The larger the number of particles sampled, the smaller the variation in the measurement of a phase's volume fraction. Because the discussion is framed in terms of cubic RVE geometries, the dimensionless cube length $\mathrm{L}^{*}$ can also be written as

$$
L^{*}=n^{(1 / 3)}
$$

Combining Eqs. (5) and (6),

$$
L^{*}=\left(\frac{p(1-p)}{\sigma_{\phi}^{2}}\right)^{(1 / 3)}
$$

Equation 7 predicts the size of an RVE given a guess $p$ for the volume fraction and the variation the researcher is willing to accept in their measurement in terms of the standard deviation $\sigma_{\square}$ of the volume fraction. Equation 7 is plotted in Fig. 1 over a complete range of possible $p$ values and several values of $\sigma_{\square}$, linearly spaced between 0.01 and 0.1 .

Examining Fig. 1 shows that, most significantly, the size of an RVE will depend on the level of precision the researcher requires. For example, if the researcher has a phase with an 
estimated volume fraction of $p=0.5$ and would like to know the true volume fraction within a standard deviation of 0.01 , an RVE of size $L^{*} \sim 14$ would be required. However, if knowing the exact volume fraction is not very important, and a standard deviation of 0.1 is tolerable, then an RVE of size $\mathrm{L}^{*} \sim 3$ will suffice. Secondly, the RVE size is dependent on the estimation of $p$. Very small and very large volume fractions can be measured with relatively small RVEs. However, to measure a moderate volume fraction with the same level of precision requires a larger RVE.

The model developed in this work is similar to a model used by Kanit et al., which also approaches the determination of a microstructural volume fraction property from a statistical sampling viewpoint.[1] In that way, the approach taken in this work for measuring volume fraction is not new, but there is a key difference in the metric which is used to define the microstructural length scale at which individual features exist. In this work, a linear dimension, the mean "particle" size, is used to define the microstructural features and the domain size via L*. Kanit et al.'s approach utilizes the mathematical concept of integral range, which provides a measurement of characteristic feature size in units of volume, rather than length. A further description of the development and definition of the integral range concept can be found in referenced work.[27] The sampling volume is then defined relative to how many of these random and independent volume units must be sampled to provide a certain desired precision. The end result from both approaches is quite similar, and is demonstrated in Kanit et al. for Voronoi mosaic structures, with results reported in Fig. 14 and Table 4 of the cited work.[1]

The advantage of the current approach is the simple interpretation and determination of the scaling unit, notably a linear feature size rather than a characteristic volume. From an experimental perspective, it is intuitively somewhat easier to observe a microstructure in 
preliminary studies, such as using SEM, and create a reasonable estimation of the characteristic particle length, as opposed to the volume, based off 2-D images. (Of course, that characteristic length could be raised to the third power to create a volumetric description of the characteristic feature size if desired). In addition, the characteristic length scaling used in this work also provides a convenient and consistent framework for determining sampling volumes for particle size and network contiguity properties, which are covered in the subsequent sections and were not covered by Kanit et al.

\subsection{Analytical Model and Results for the Estimation of Particle Size Distribution RVE Size}

Although in the previous formulation all particles of all phases were assumed to be equal in size, in reality there is some distribution of particle sizes (and some mean) for each phase. To describe this distribution of particle sizes, it is necessary to sample a sufficient number of particles such that the full span of possible sizes has been included in the sample. The problem is one of statistical sampling which asks how many items must be sampled from a population in order for the sample distribution (and therefore the sample statistics) to be representative of the true population distribution. In this model, the sample mean particle size is used as the sample statistic. A sample size which consistently produces the correct sample mean can only be achieved if the sample itself includes a representative and consistent distribution of particle sizes. Assume $\sigma_{\text {pop,i }}$ is the standard deviation of particle sizes for the entire population of particles of phase $i$ throughout the total material domain. The central limit theorem of statistics states that if many (approaching infinity) samples of phase $i$ of $n_{i}$ particles each are taken from a population, and the mean particle size of each sample set is computed, the sample means will follow a normal distribution with the same mean as the population and a standard deviation given by 


$$
\sigma_{i}=\frac{\sigma_{p o p, i}}{\sqrt{n}_{i}}
$$

where $\sigma_{\mathrm{i}}$ is the standard deviation in the measurement of mean particle size when many samples of $n_{i}$ particles each are taken from a population much larger than the sample. Rearranging equation (8) leads to,

$$
n_{i}=\left(\frac{\sigma_{p o p, i}}{\sigma_{d e s, i}}\right)^{2}
$$

where the standard deviation of the sample means has now been relabeled as the researcher's desired standard deviation $\sigma_{\text {des,i }}$. This is the variation the researcher is willing to tolerate in the computation of the mean particle size of phase $i$. If the researcher does not need to know the particle sizes with much certainty, $\sigma_{\text {des,i }}$ will be relatively large, and therefore the number of phase $i$ particles which need to be included in the sample is relatively small. Conversely, if the researcher would like to know the sizes of phase $i$ particles with a high level of precision, the acceptable standard deviation $\sigma_{\text {des,i }}$ is relatively small, and a large number $n_{i}$ of particles will need to be sampled.

It is realized that phase $i$ particles account for only a fraction of the total particles in the system, approximately by

$$
n_{i}=p_{i} n
$$

where $p_{i}$ is the estimated volume fraction of phase $i$, and $n$ is the total number of particles in the system. By combining Eqs. (1), (9), and (10),

$$
L^{*}=\frac{\frac{\text { pop }}{(2 / 3)} \div}{p^{(1 / 3)}}=\frac{(\text { SDratio })^{(2 / 3)}}{p^{(1 / 3)}}
$$


Equation (11) provides an analytical expression for RVE size (where the subscript $i$ has been dropped for clarity) as a function of two estimated parameters and one constraint. The estimated parameters, $p$ and $\sigma_{\mathrm{pop}}$ represent the researcher's estimates of the volume fraction of the phase and the standard deviation of particle sizes they expect across the total material domain. Neither is known a priori, but can be approximated based on fabrication conditions, preliminary pilot studies, or $2 \mathrm{D}$ micrographs. The ratio of the standard deviations in the numerator will be referred to as the SD ratio. Plots of $\mathrm{L}^{*}$ vs. $p$ for a range of SD ratios between 1 and 10 is shown in Fig. 2.

As expected the RVE size depends on the level of precision the researcher requires. A large SD ratio (indicative of a wide variation of particle sizes in the population and/or a small acceptable variation in the results) requires a larger RVE to sample enough particles. A small SD ratio (indicative of minor variations in particle sizes and/or a large acceptable variation in the results) permits a smaller RVE to be used. Secondly, phases that account for a small volume fraction generally have substantial separation between their constituent particles, and therefore a larger total sample of the material needs to be analyzed to capture a sufficient number of the particles. A phase accounting for a high fraction of the volume, on the other hand, has particles located in close proximity to one another within the sample, so less total material needs to be sampled to obtain the same number of particles of that phase.

\subsection{Simulation Model for Network Contiguity}

The development of a descriptive model for network contiguity is somewhat more difficult than the previous descriptions. In the previous sections, simplified analytical models were developed for measurement of volume fraction and particle size distribution due to the 
inherent local nature of those measurements. However, the description of network contiguity is complicated by the fact that the network depends on the long range order of the structure. The contribution of a single particle to a transport process within a network cannot be evaluated considering that particle alone; it is necessary to look at the amount and spatial distribution of the other particles to which it is connected (or not connected).

\subsubsection{Percolation Theory}

Guidance for the analysis of network properties can be found in existing analytical and numerical work in percolation theory.[28-30] In general, percolation theory is concerned with describing the long-range connectedness of particle networks in an infinite domain. The discussion of percolation theory in this paper is far from comprehensive, and is focused only on the connection of theory with real data from an experimental characterization point of view. First, some general concepts must be introduced. Particles within an infinite domain can exist in one of two possible states:

1) The particle can belong to a particle cluster which is isolated and finite in size. The size of the cluster can range from 1 particle to the theoretical size of infinity minus one.

2) The particle can be a part of a cluster which extends in size to infinity. Such an infinite cluster will be called a percolating network, or simply a network.

A percolation threshold can be defined, which serves as a cut-off differentiating systems which contain only isolated clusters from systems which contain an infinite network. This percolation threshold is typically defined in terms of a critical volume fraction of a given phase, denoted $\square_{\mathrm{c}}$ :

$\square<\square_{\mathrm{c}} \quad$ Only isolated clusters exist 


\section{$>\square_{\mathrm{c}} \quad$ An infinite network exists}

A number of works have aimed at predicting the percolation behavior in composite materials in two or three dimensions.[23,25,26,31-34] Typically, the composite structure is simplified to an easily tractable geometrical model with a given type of packing (such as simple cubic, body-centered cubic, random, etc. and defined by particle coordination number, Z) of a binary system of spheres. Using the packing structure and the sizes of particles comprising the various phases, analytical expressions have been developed to predict the percolation threshold of each phase.

Results from Bouvard et al. suggest that although the value of the percolation threshold $\square_{\mathrm{c}}$ can vary, for example when particle size ratio is varied, the general percolation behavior in the vicinity of the threshold remains similar (see Fig. 3 in Bouvard).[31] This behavior is depicted in Fig. 3. A phase below the percolation threshold contains only isolated clusters, and if more particles of that phase are added to the system and the volume fraction increased, the size of the isolated clusters would grow, but they would remain distinct from one another (regime 1). When the volume fraction of the phase is increased to near the threshold, large clusters of particles exist, and the subtle yet significant difference between non-percolating and percolating networks will be due to a seemingly minor local change in particle placement which has longrange implications. A phase just above the percolation threshold forms a connected and infinite network by bridging the gaps between some of the existing isolated clusters. While there is a very likely chance that such an infinite network exists above the threshold, there can still be many isolated clusters which are not a part of the network. Therefore, the particles would have some probability of being connected to the infinite network, and some probability of being part of a finite isolated cluster (regime 2). To continue the process, if more particles are added and 
the volume fraction increased farther, more and more of the isolated clusters become connected to the network, and a smaller fraction remain isolated (regime 3). If the process of adding particles is continued to the limiting case of occupying the entire volume, $100 \%$ of the particles are connected to the infinite network and there are no isolated clusters of any size remaining.

\subsubsection{Simulation Methods}

To describe network contiguity in the RVE context, analytical theory will be complemented by a simulation approach. Simulation is able to capture the randomness and longrange effects that impact the structure when the composition is near the percolation threshold, which is critical in the cases where the "averaged" behavior predicted by the analytical theory is not sufficiently descriptive. Repeating simulations many times can provide estimates of percolation thresholds as well as the variations in percolation behavior from sample to sample. Most importantly, within the framework of the RVE concept, simulations can also provide insight into how these descriptions of the network change as a function of volume size $\mathrm{L}^{*}$.

A number of researchers have previously performed computer simulations to describe percolation. Most work has focused on determining the percolation threshold itself rather than the contiguity and size-dependence of the network phenomenon. For example, a number of past simulation studies have shown the threshold for site percolation on a simple cubic lattice is given by a site fraction $f_{i}=0.3116 .[35-38]$ However, if the sites are assumed to be occupied by spherical particles rather than cubic sites, which is a more realistic approximation of real materials, conversion from the site fraction $f_{i}$ to the volume fraction $\square_{\mathrm{i}}$ of the spherical particles is simply given by a geometrical adjustment

$$
\phi_{i}=f_{i} \frac{\pi}{6}
$$


Thus the critical volume fraction on a simple cubic lattice is about $\square_{\mathrm{c}}=0.16$, which is quite similar to the value of $\square_{\mathrm{c}}=0.187$ reported by Kuo and Gupta for percolation of randomlyplaced spheres in a continuous medium.[32] In fact, the critical volume fraction in 3-D has been shown to be relatively insensitive to the packing structure in the case of monosized spherical particles.[23,31,39]

In addition to calculating the percolation threshold, at least a few studies have explicitly reported the size of the simulation domain relative to the particle size. Powell et al. found that when the composition of the material is near the percolation threshold, an $\mathrm{L}^{*}$ value greater than 20 is needed to reliably capture the transition from non-percolating to percolating structures.[39] When the composition is not near the percolation threshold, an $\mathrm{L}^{*}$ of 20 or less may be appropriate, more so the farther the composition is from the threshold.

Bouvard et al. performed more extensive simulation studies, and found that when particle size was made too large (akin to $\mathrm{L}^{*}$ too small), significant scatter was present in the results when the material composition was near the percolation threshold.[31] The authors attributed this effect to too few of the large particles being present in the domain to provide a good statistical measure of the network. When particles were made smaller, and thus $\mathrm{L}^{*}$ values larger (in the vicinity of $\mathrm{L}^{*}=40$ ), the data was more consistent. However, for most cases the authors did not analyze replicate volumes at a given size and composition, so they were not able to report a variation in results due to random statistical variations in particle placement. In addition, for fixed particle size the authors did not simulate domains of different sizes to determine the impact of the total simulation volume on the percolation behavior.

Suzuki and Oshima presented a percolation model for a multi-component mixture of spherical particles aimed at estimating particle coordination numbers.[34] Computer simulation 
with randomly-generated packed sphere structures with $\mathrm{L}^{*}$ ranging from 7.5 to 15 was used to confirm the validity of the model. However, the study was not explicitly interested in variation of percolation behavior with domain size, and the overall contiguity of particles of a certain type was not reported.

Simulation studies performed by Acharyya and Stauffer as well as Jan and Stauffer examined percolation behavior of networks with the composition fixed at the percolation threshold.[37,38] The authors report the fraction of percolating domains, which they call the critical spanning probability, as well as cluster sizes. The results are found to depend on boundary conditions, the dimensions of the lattice, as well as domain size. In 3-D, lattice dimensions in excess of $\mathrm{L}^{*}=1000$ were examined with results appearing to reach asymptotic values at around $\mathrm{L}^{*}=100$, indicating volume independence.

Additional simulations have been performed by several authors with the intention of describing the microstructure of a particular device, namely solid oxide fuel cell composite electrodes.[19,21,22,25] Bertei et al. found that using simulation domains with dimensions $L^{*}=16 \times 16 \times 48$ generally gave less than $2 \%$ variation amongst replicate random volumes for a variety of investigated properties including contiguity, tortuosity, mean pore size, and triple phase boundary length. The authors also observed the same percolation behavior as shown in Fig. 3 (presented as Fig. 4 in Bertei et al.) for the pore phase, albeit at a lower volume fraction owing to the small "particle" size of the pores. Kenney et al. used cubic simulation domains with $\mathrm{L}^{*}$ equal to approximately 25 for measuring properties on structures above the percolation threshold. However, for all measurements the authors averaged results over 20 random replicate structures, and did not report the variation between individual replicates. Lastly, simulations of electrode microstructures by Cai et al. gave consistent measurements (within an acceptable 
variation of $10 \%$ ) of percolated triple phase boundary density for $L^{*}>7.5$. The simulations were performed for three interconnected phases with volume fractions of $0.215,0.350$, and 0.435 .

The simulations presented in this work build upon those previously reported. In particular, the current simulations aim to analyze the variations in network percolation for replicate cubes of the same size and same composition, as well as cubes of varying size. Studying these variations will help provide an estimate of the size $\mathrm{L}^{*}$ required to accurately describe the overall material network properties.

The simulated structures used in this work are generated in the following manner. Particles of all phases are assumed to be non-overlapping but touching mono-sized spheres and occupy sites on a simple cubic lattice. Site percolation, as opposed to bond percolation, is considered in this work. The lattice itself is a cube of prescribed dimension $\mathrm{L}^{*}$ on a side, and contains 2 phases: the phase of interest with a site fraction of $f_{i}$, and the "other" phases grouped collectively as not belonging to the phase of interest with site fraction $\left(1-f_{i}\right)$. This approach implicitly assumes that the phase of interest and the other phases consist of particles of a similar size, thereby excluding drastic disparities in length scales. The cubic volume is considered one lattice site at a time, and each lattice point has a probability $f_{i}$ of being labeled as a particle of the phase of interest. After each lattice site has been considered, a number $n_{i}$ of them have been labeled as particles of the phase of interest, occupying approximately, but not necessarily exactly, a fraction $f_{i}$ of the total possible lattice sites.

Simulation domains are generated varying $\mathrm{L}^{*}$ between 6 and 100, and $f_{i}$ between 0.02 and 0.9. For each combination of cube size $\mathrm{L}^{*}$ and composition $f_{i}, 30$ random replicate volumes are generated. In each replicate, the placement of spheres occurs at random lattice locations, and therefore the replicates are all different (and can conceptually be thought of as different 
experimental samples extracted from the same total material domain), accounting for the variations in long-range structure that can be seen even in samples with the same size and composition.

Each cube is analyzed using a painting algorithm to determine its contiguity. The algorithm follows a similar procedure as the Hoshen-Kopelman algorithm and starts by searching iteratively through the volume for a particle of the phase of interest which has not yet been marked.[40] Once found, that particle is marked, and its nearest neighbor lattice points (6 neighbors exist in 3-D) are examined to see if any of them contain unmarked particles. If so, those particles are marked, and their neighbors are examined, and so on. The procedure continues until all particles connected to the single original particle have been given the same marker. The search through the volume then continues until a new unmarked particle is found. That particle is then marked with a new marker and its neighbors examined in the same fashion. The algorithm terminates when all particles have been marked.

Two implementations of boundary conditions have been investigated and are referred to as type 1 and type 2 . Type 1 uses free boundary conditions, which are physically equivalent to symmetry boundaries on all 6 sides of the cube. For type 2, one coordinate direction is arbitrarily chosen as the transport direction, and periodic boundaries are applied in the remaining two directions. The two cases are presented because, although it has seen some discussion in the literature, there is not yet a consensus on the most appropriate type of boundary condition when measuring pathway-type properties on experimental RVEs.[15,18,41,42] After the labeling algorithm is complete, all particles connected to a common cluster have the same marker, while separate clusters have different markers. 
Every cluster is then examined and classified based upon its connectedness to the cube boundaries. For type 1 the classifications are:

- A cluster intersecting all 6 faces of the cube is considered contiguous.

- A cluster that does not intersect any of the cube faces is an isolated cluster.

- A cluster that intersects at least 1 , but less than 6 , of the cube faces is a dead-end cluster. For type 2:

- A cluster intersecting the 2 faces of the cube in the transport direction is considered contiguous.

- A cluster which does not intersect either of the faces in the transport direction is isolated.

- A cluster which intersects only one of the faces in the transport direction is a dead-end cluster.

For both cases, the term contiguity refers to the fraction of particles connected to a contiguous (percolating) cluster based on the definitions above.

Before discussion of the results, it is important to recall that each cube is conceptually considered to be an experimental sample extracted from within a much larger domain. The domain, presumably the entire device with some large but finite size, may contain clusters which are contiguous, isolated, or connected only at certain boundaries as dead-ends. However, when a sample is extracted from an arbitrary location within the domain, new boundaries are artificially created and existing clusters are truncated. Therefore, there exists the possibility for the clusters within the sample to be categorized incorrectly. For example, a cluster which may be large but isolated in the total domain may, by chance, happen to intersect all faces of a small sample volume and be incorrectly categorized as contiguous. Conversely, a contiguous network in the total domain may intersect a sample boundary only partially, leading to the incorrect 
categorization of "dead-end" particles. The prevalence of these categorization errors will depend on the sample size as well as its composition relative to the percolation threshold. In general, large samples and/or compositions away from the percolation threshold will have fewer errors than small samples and/or compositions near the percolation threshold. This idea follows intuitively from the earlier discussion on cluster size.

\subsubsection{Contiguity Simulation Results and Discussion}

Simulation results are presented in Fig. 4 for several different values of L*. Results are reported in terms of the contiguity as a function of the volume fraction relative to the percolation threshold, which as discussed previously is approximately $\square_{c}=0.16$ for monosized spherical particles in 3-D. Panels (a)-(d) are the results for type 1 boundaries, and panels (e)-(h) are for type 2 boundaries. On each plot, a black line is included to show the expected behavior in the limit of an infinite domain. This was approximated by fitting a curve through the data points for the domain of $L^{*}=100$, as the scatter was minimal and thus the data seemed to be approaching the infinite limit. The black curves are almost exactly identical for the two types of boundary conditions. It is apparent that for the type 1 boundaries, at small $\mathrm{L}^{*}$ the data seem to lie beneath the black curve, while for the type 2 boundaries they have a more even spread around the black curve. This can be explained considering that for type 1 boundaries, isolated particles located on the domain boundary will never be labeled as connected, and therefore the fraction of contiguous particles is underestimated. Conversely, for type 2 boundaries, particles located on a domain boundary orthogonal to the transport direction have some chance of being connected to the structure on the opposite side of the domain via periodicity. Therefore, in that case the 
placement of the particle on the boundary is no different than any other location in the interior of the volume, and a greater measure of contiguity is possible.

The effects of sample size can be described using Fig. 4. For $L^{*}=6$, substantial variations in the contiguity are observed close to the percolation threshold for both types of boundaries. There are numerous instances where the volume fraction of the phase is below the threshold, yet the cube displays percolating behavior, and there are also many instances where the volume fraction is greater than the threshold yet cubes display completely non-percolating behavior. Both of these cases represent errors introduced by the small sample size near the percolation threshold. Furthermore, the spread of data in the horizontal direction provides evidence of the difficulty in interpreting experimental results. This is largely because when a real sample is examined experimentally, the percolation threshold of a phase is generally not known, so it is also unknown whether the phase is above or below the threshold. So for example, if a cube of $L^{*}=6$ is measured experimentally to have a contiguity of 0.8 , then that phase could actually have a composition ranging from just below to well above the percolation threshold. Likewise, if a single cube is measured to have a contiguity of 0 , it could correspond to a material with a contiguity which is actually zero, or could be as large as roughly $90 \%$.

Clearly the measurement of a single small volume is not useful to the experimentalist for either type of boundary. However, measurements taken on replicate small cubes could be beneficial and provide more evidence as to the material's composition relative to the percolation threshold. This could be useful, for example, in circumstances where experimentally obtaining a large cube may not be possible due to limited total domain size. For this type of analysis, if the researcher measures a single cube of $L^{*}=6$ and obtains a contiguity of 0 , but then measures several other samples and gets results in the range of $0.7-0.9$, it could be reasonably concluded 
that the sample is almost certainly above the percolation threshold, and most particles are connected to a percolating network. The true value of contiguity is likely somewhere in the range of $0.7-0.9$, and the first measurement is probably an erroneous measurement. However, in general, averaging the results from many replicate small cubes will not produce exactly the same value of contiguity as a single measurement of a larger, volume independent cube, and is therefore not quantitatively correct. In addition, any individual measurement of a small cube is in itself still not representative. But the utility of this approach is that it can provide a more educated guess of the composition of the material relative to the percolation threshold when limited experimental samples sizes are necessary.

Interpretation of the results is much more straightforward when large volumes are used. As percolation theory has been developed in the hypothetical context of an infinite domain, as the simulated or experimental sample size is made very large, the results obtained will approach the infinite limit and become independent of boundary conditions. This behavior can be seen for $\mathrm{L}^{*}=100$. For a given measured contiguity, there is no longer any ambiguity of where the true material lies relative to the percolation threshold. A measured contiguity value of 0.5 , for example, can only correspond to a phase which has a volume fraction about 0.01 greater than the percolation threshold in the case of the simple cubic structure. In addition, a measured contiguity of 0 is certain to mean the material is at or below the percolation threshold, and a measured contiguity of 1 (or nearly 1) is certain to mean the material is well above the percolation threshold and nearly completely contiguous.

It is therefore up to the researcher how much uncertainty is tolerable. If one would just like to know whether the phase is in percolation regime 1, 2, or 3, described in Fig. 3, then it might be sufficient to simply measure several small samples, say of $L^{*}<10$. However, if the 
researcher is interested in a more exact and quantitative measure of the fraction of contiguous particles, or would like a single cube which displays representative behavior for subsequent quantitative modeling for example, a small volume will not suffice and a larger cube must be examined. In this case, the closer the composition to the percolation threshold and the higher the accuracy desired, the larger the cube must be. For example, if a researcher suspects or knows their material to be well above the percolation threshold, this could be confirmed by making measurements on a cube of $\mathrm{L}^{*}=10$. For a cube of $\mathrm{L}^{*}=10$, if the results indicate a very high contiguity, say greater than $95 \%$, it can be confirmed using Fig. 4 that the material is in fact well above the percolation threshold with a high contiguity. But alternatively if the researcher suspects their material is close to the percolation threshold, $\mathrm{L}^{*}=10$ will not be sufficient as a significant spread of results can occur for this volume size. In this case, a larger volume, perhaps $\mathrm{L}^{*}>30$, will be necessary to produce the desired accuracy.

From an experimental perspective, the applicability of each of the types of boundary conditions must also be considered. In reality, no physical sample would be expected to display a periodic or repeated symmetric structure. Therefore, the use of either in characterization of real materials is an additional approximation of the larger structure, and will be discussed further in Part 2 of this work.

\section{Outlook for RVE Models}

While only three morphological properties were considered explicitly in this work, hypotheses are presented for two additional properties. First, accurate measurement of specific surface area (or in the case of a multi-phase system, interfacial contact area or triple phase boundary length) is expected to follow the accurate measurement of volume fraction and particle 
size. It is intuitive to infer that, in order to quantify the interfacial characteristics between two phases, each phase itself must be accurately quantified. If the description of volume fraction or particle sizes of a given phase contains a large amount of uncertainty, so should the description of that phase's contacts with its neighboring phases.

In addition, the required RVE size for measurement of network tortuosity is expected to follow a similar trend as that of network contiguity. Tortuosity, which is the ratio of an effective path length divided by nominal path length, is written as:

$$
\tau=\frac{L_{\text {eff }}}{L}
$$

A number of researchers have implemented algorithms for measuring tortuosity on real structures.[13,15,42-44] The methods broadly fall into two categories: effective diffusion-type simulations and geometric pathway measurements, which are discussed in detail by ChenWiegart et al.[43] Equation 13 represents an expression of the geometric, or "path length" tortuosity. However, for real structures, tortuosity is commonly determined by the diffusionbased approach, in which an apparent tortuosity is responsible for the difference in diffusion capabilities (or losses) between a real structure and a theoretical structure of straight channels. In this way, this method accounts for geometrical as well as constrictive effects due to diffusion losses in "bottleneck" structures. In both cases, the measurement of the tortuosity will depend first and foremost on having a structure which is representative of the contiguity of the network. In this sense, the tortuosity measurement can be thought of as a more detailed description; while the network contiguity provides information on whether or not a network is connected, the tortuosity provides information as to how specifically it is connected and the geometric effectiveness of those connections from a transport perspective. Therefore, it is hypothesized 
that an RVE for tortuosity measurements must be at least as large as an RVE for the contiguity measurement as described above.

\section{Conclusions}

This paper considers the microstructural characterization of particle-like composite materials with relatively uniform feature sizes and randomly distributed particles of various phases. The accurate measurement of microstructural properties is found to depend, in general, on the composition of the material, the property of interest, and the level of precision the researcher requires. Simple geometric models were presented to describe the measurement of properties for these types of materials as a function of the measurement volume size, described in terms of a dimensionless length $\mathrm{L}^{*}$. Analytical closed-form equations are used for the prediction of $\mathrm{L}^{*}$ for volume fraction and particle size properties, while a simulation approach was used to examine network contiguity. Accurate characterization of contiguous structures was found to depend strongly on the proximity of a phase to its percolation threshold. Comparison of the models to experimental data is presented in Part 2 of this work.

Acknowledgements

Financial support from an Energy Frontier Research Center on Science Based Nano-Structure

Design and Synthesis of Heterogeneous Functional Materials for Energy Systems (HeteroFoaM Center) funded by the U.S. Department of Energy, Office of Science, Office of Basic Energy Sciences (Award DE-SC0001061) and the National Science Foundation (Award CBET1134052) are gratefully acknowledged. Simulations of microstructural networks were performed 
on the HORNET cluster at the University of Connecticut's Booth Engineering Center for Advanced Technology.

References

[1] Kanit, T., Forest, S., Galliet, I., Mounoury, V., Jeulin, D., 2003, "Determination of the size of the representative volume element for random composites: statistical and numerical approach," Int.J.Solids Structures, 40(13-14), pp. 3647-3679.

[2] Gitman, I. M., Askes, H., Sluys, L. J., 2007, "Representative volume: Existence and size determination," Eng.Fract.Mech., 74(16), pp. 2518-2534.

[3] Evesque, P., 2000, "Fluctuations, correlation and representative elementary Volume (REV) in granular materials," Poudres and Grains, 11, pp. 6-17.

[4] Ostoja-Starzewski, M., 2006, "Material spatial randomness: From statistical to representative volume element," Prob.Eng.Mech., 21(2), pp. 112-132.

[5] Stroeven, M., Askes, H., Sluys, L., 2004, "Numerical determination of representative volumes for granular materials," Comput.Methods Appl.Mech.Eng., 193(30), pp. 3221-3238.

[6] Trias, D., Costa, J., Turon, A., Hurtado, J., 2006, "Determination of the critical size of a statistical representative volume element (SRVE) for carbon reinforced polymers," Acta materialia, 54(13), pp. 3471-3484.

[7] Vinogradov, O., 2001, "On a representative volume in the micromechanics of particulate composites," Mechanics of composite materials, 37(3), pp. 245-250.

[8] Wilson, J. R., Cronin, J. S., Barnett, S. A., 2011, "Linking the microstructure, performance and durability of Ni-yttria-stabilized zirconia solid oxide fuel cell anodes using threedimensional focused ion beam-scanning electron microscopy imaging," Scr.Mater., 65(2), pp. 67-72.

[9] Cronin, J. S., Wilson, J. R., Barnett, S. A., 2011, "Impact of pore microstructure evolution on polarization resistance of Ni-Yttria-stabilized zirconia fuel cell anodes," J.Power Sources, 196(5), pp. 2640-2643.

[10] Ender, M., Joos, J., Carraro, T., Ivers-Tiffée, E., 2012, "Quantitative Characterization of LiFePO4 Cathodes Reconstructed by FIB/SEM Tomography," J.Electrochem.Soc., 159(7), pp. A972-A980. 
[11] Grew, K. N., Chu, Y. S., Yi, J., Peracchio, A. A., Izzo Jr., J. R., Hwu, Y., De Carlo, F., Chiu, W. K. S., 2010, "Nondestructive nanoscale 3D elemental mapping and analysis of a solid oxide fuel cell anode," J.Electrochem.Soc., 157(6), pp. B783-B792.

[12] Shearing, P., Bradley, R., Gelb, J., Tariq, F., Withers, P., Brandon, N., 2012, "Exploring microstructural changes associated with oxidation in Ni-YSZ SOFC electrodes using high resolution X-ray computed tomography," Solid State Ionics, 216 pp. 69-72.

[13] Shearing, P. R., Howard, L. E., Jørgensen, P. S., Brandon, N. P., Harris, S. J., 2010, "Characterization of the 3-dimensional microstructure of a graphite negative electrode from a Liion battery," Electrochem. Commun., 12(3), pp. 374-377.

[14] Romero, P., Masad, E., 2001, "Relationship between the representative volume element and mechanical properties of asphalt concrete," J.Mater.Civ.Eng., 13(1), pp. 77-84.

[15] Laurencin, J., Quey, R., Delette, G., Suhonen, H., Cloetens, P., Bleuet, P., 2012, "Characterisation of Solid Oxide Fuel Cell Ni-8YSZ substrate by synchrotron X-ray nanotomography: from 3D reconstruction to microstructure quantification," J.Power Sources, 198(0), pp. 182-189.

[16] Metcalfe, C., Kesler, O., Rivard, T., Gitzhofer, F., Abatzoglou, N., 2010, "Connected threephase boundary length evaluation in modeled sintered composite solid oxide fuel cell electrodes," J.Electrochem.Soc., 157(9), pp. B1326-B1335.

[17] Lombardo, J. J., Ristau, R., Harris, W. M., Chiu, W. K. S., 2012, "Focused Ion Beam Preparation of Samples for X-ray Nanotomography," J. Synchrotron Radiat., 19 pp. 789-796.

[18] Joos, J., Ender, M., Carraro, T., Weber, A., Ivers-Tiffée, E., 2012, "Representative volume element size for accurate solid oxide fuel cell cathode reconstructions from focused ion beam tomography data," Electrochim.Acta, 82 pp. 268-276.

[19] Kenney, B., Valdmanis, M., Baker, C., Pharoah, J. G., Karan, K., 2009, "Computation of TPB length, surface area and pore size from numerical reconstruction of composite solid oxide fuel cell electrodes," J.Power Sources, 189(2), pp. 1051-1059.

[20] Bertei, A., Nucci, B., Nicolella, C., 2013, "Microstructural modeling for prediction of transport properties and electrochemical performance in SOFC composite electrodes," Chemical Engineering Science, 101 pp. 175-190.

[21] Cai, Q., Adjiman, C. S., Brandon, N. P., 2011, "Modelling the 3D microstructure and performance of solid oxide fuel cell electrodes: Computational parameters," Electrochim.Acta, 56(16), pp. 5804-5814.

[22] Berson, A., Choi, H., Pharoah, J. G., 2011, "Determination of the effective gas diffusivity of a porous composite medium from the three-dimensional reconstruction of its microstructure," Physical Review E, 83(2), pp. 026310. 
[23] Costamagna, P., Costa, P., Antonucci, V., 1998, "Micro-modelling of solid oxide fuel cell electrodes," Electrochim.Acta, 43(3-4), pp. 375-394.

[24] Cannarozzo, M., Borghi, A. D., Costamagna, P., 2008, "Simulation of mass transport in SOFC composite electrodes," J.Appl.Electrochem., 38(7), pp. 1011-1018.

[25] Bertei, A., Nicolella, C., 2011, "Percolation theory in SOFC composite electrodes: Effects of porosity and particle size distribution on effective properties," J.Power Sources, 196(22), pp. 9429-9436.

[26] Chen, D., Lin, Z., Zhu, H., Kee, R. J., 2009, "Percolation theory to predict effective properties of solid oxide fuel-cell composite electrodes," J.Power Sources, 191(2), pp. 240-252.

[27] G. Matheron, Estimating and choosing, Springer; 1989.

[28] A. Bunde, S. Havlin, Fractals and disordered systems, Springer-Verlag New York, Inc.; 1991.

[29] Grimmett, G., 1999, "Percolation", volume 321 of "Grundlehren der mathematischen Wissenschaften".

[30] A. Aharony, D. Stauffer, Introduction to percolation theory, Taylor \& Francis; 2003.

[31] Bouvard, D., Lange, F., 1991, "Relation between percolation and particle coordination in binary powder mixtures," Acta metallurgica et materialia, 39(12), pp. 3083-3090.

[32] Kuo, C., Gupta, P. K., 1995, "Rigidity and conductivity percolation thresholds in particulate composites," Acta metallurgica et materialia, 43(1), pp. 397-403.

[33] Nan, C., Shen, Y., Ma, J., 2010, "Physical properties of composites near percolation," Annual Review of Materials Research, 40 pp. 131-151.

[34] Suzuki, M., Oshima, T., 1983, "Estimation of the co-ordination number in a multicomponent mixture of spheres," Powder Technol, 35(2), pp. 159-166.

[35] Deng, Y., Blöte, H. W., 2005, "Monte Carlo study of the site-percolation model in two and three dimensions," Physical Review E, 72(1), pp. 016126.

[36] Wang, J., Zhou, Z., Zhang, W., Garoni, T. M., Deng, Y., 2013, "Bond and site percolation in three dimensions," Physical Review E, 87(5), pp. 052107.

[37] Jan, N., Stauffer, D., 1998, "Random site percolation in three dimensions," International Journal of Modern Physics C, 9(02), pp. 341-347.

[38] Acharyya, M., Stauffer, D., 1998, "Effects of boundary conditions on the critical spanning probability," International Journal of Modern Physics C, 9(04), pp. 643-647. 
[39] Powell, M., 1979, "Site percolation in randomly packed spheres," Physical Review B, 20(10), pp. 4194.

[40] Hoshen, J., Kopelman, R., 1976, "Percolation and cluster distribution. I. Cluster multiple labeling technique and critical concentration algorithm," Physical Review B, 14(8), pp. 34383445 .

[41] Joos, J., Carraro, T., Weber, A., Ivers-Tiffée, E., 2011, "Reconstruction of porous electrodes by FIB/SEM for detailed microstructure modeling," J.Power Sources, 196(17), pp. 7302-7307.

[42] Grew, K. N., Peracchio, A. A., Joshi, A. S.,Izzo Jr., J. R., Chiu, W. K. S., 2010, "Characterization and analysis methods for the examination of the heterogeneous solid oxide fuel cell electrode microstructure. Part 1: Volumetric measurements of the heterogeneous structure," J.Power Sources, 195(24), pp. 7930-7942.

[43] Chen-Wiegart, Y.-C. K., DeMike, R., Erdonmez, C., Thornton, K., Barnett, S. A., Wang, J., 2014, "Tortuosity characterization of 3D microstructure at nano-scale for energy storage and conversion materials," J.Power Sources, 249 pp. 349-356.

[44] Cronin, J. S., Chen-Wiegart, Y.-C. K., Wang, J., Barnett, S. A., 2013, "Three-dimensional reconstruction and analysis of an entire solid oxide fuel cell by full-field transmission X-ray microscopy," J.Power Sources, 233 pp. 174-179.

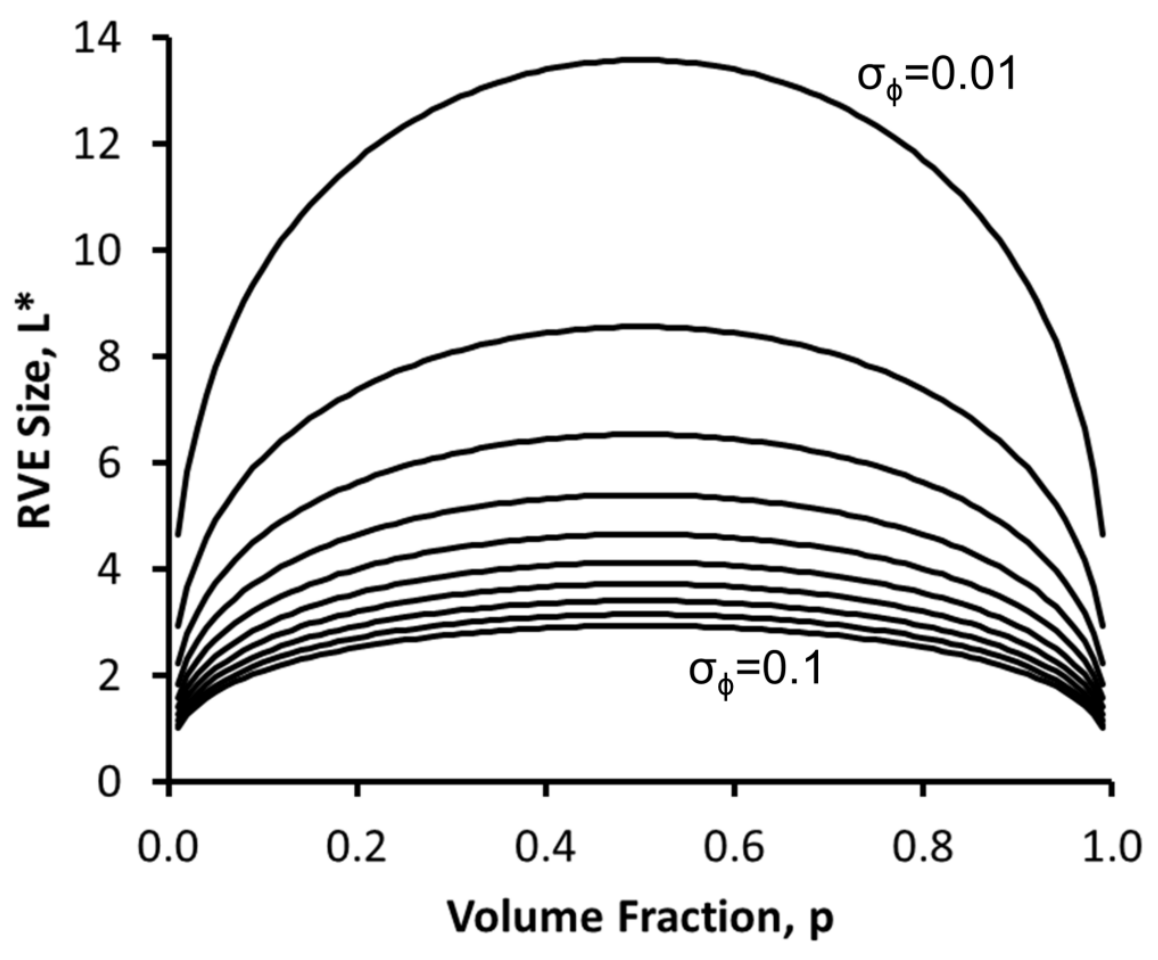


Figure 1: Analytical predictions for RVE size required for measurement of volume fraction. The required RVE size is a function of the researcher's estimate of the volume fraction, $p$, and the tolerable standard deviation in the results $\sigma_{\square}$. 


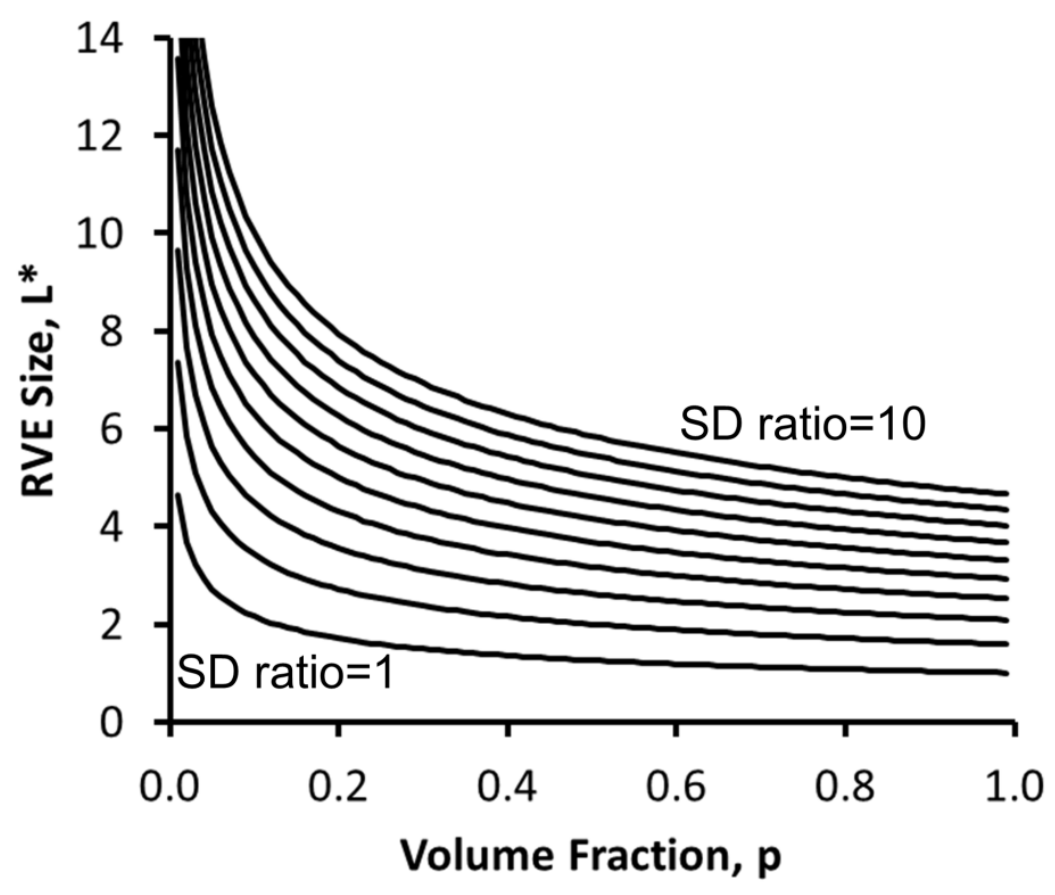

Figure 2: Analytical predictions for RVE size required for measurement of particle sizes. The required RVE size is a function of the researcher's estimate of the phase's volume fraction $p$, the estimate of the standard deviation of particle sizes which exist in the sample $\sigma_{\mathrm{pop}}$, and the tolerable standard deviation in the measurement of the mean diameter $\sigma_{\text {des. }}$. 


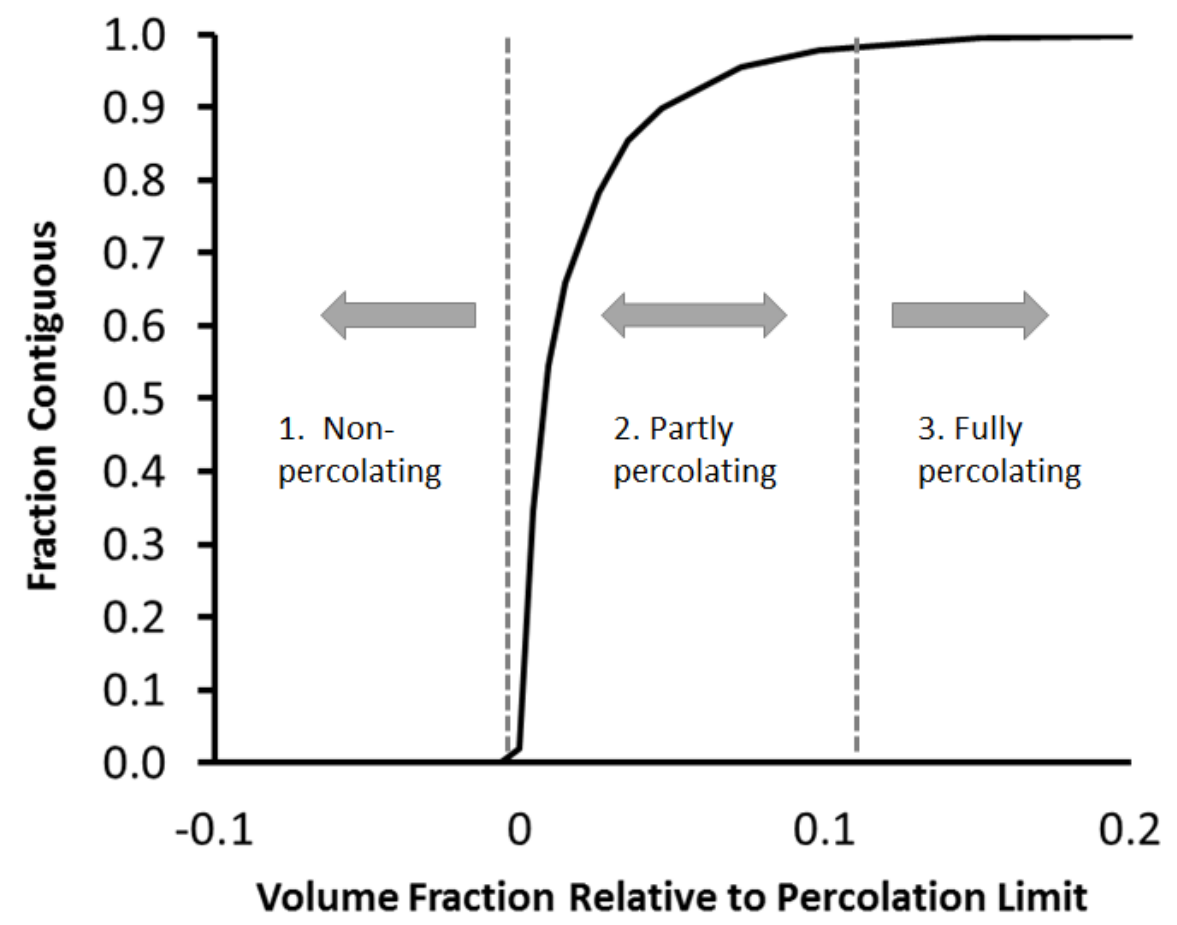

Figure 3: Percolation behavior can be qualitatively described in terms of three regimes. The results shown here are for site percolation of spherical particles on a simple cubic lattice in 3-D. 

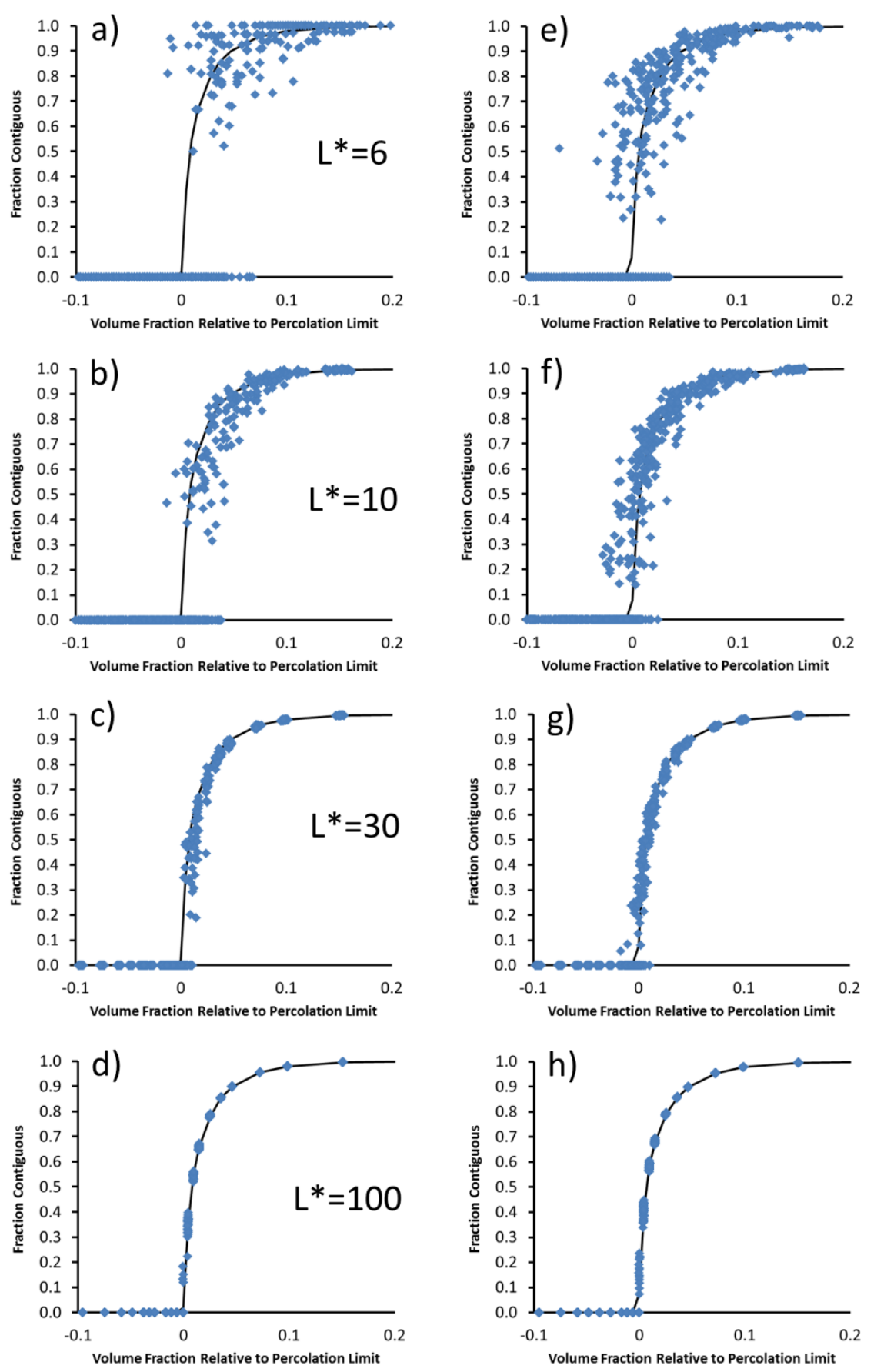

Figure 4: Results for contiguity of simulated structures. Results are shown for four different cube sizes, $L^{*}=6,10,30$, and 100 . (a)-(d) are obtained using type 1 boundary conditions with no preference to transport direction, while (e)-(h) are obtained using type 2 boundaries by assuming a primary transport direction and implementing periodic boundary conditions in the orthogonal directions. 